(2) Open Access Full Text Article

\title{
Impact of Caregiving Burden on Quality of Life of Caregivers of COPD Patients: The Chain Mediating Role of Social Support and Negative Coping Styles
}

\author{
Mo Yi (D) \\ Di Jiang ${ }^{2}$ \\ Yuanmin Jia' \\ Wei $\mathrm{Xu}^{3}$ \\ Haixia Wang' \\ Yizhang $\mathrm{Li}^{\prime}$ \\ Zeyi Zhang (D') \\ Jingjing Wang $\mathbb{D}^{\prime}$ \\ Ou Chen'
}

'School of Nursing and Rehabilitation, Cheeloo College of Medicine, Shandong University, Jinan, Shandong, 2500I2, People's Republic of China; ${ }^{2}$ Peking Union Medical College Hospital, Beijing, 100730, People's Republic of China; ${ }^{3}$ Department of Psychology, Shanghai Normal University, Shanghai, 200234, People's Republic of China
Correspondence: Ou Chen

School of Nursing and Rehabilitation, Cheeloo College of Medicine, Shandong University, 44\# Wenhua xi Road, Lixia District, Jinan City, Shandong Province,

People's Republic of China

Tel +8653188382000

Email chenou@sdu.edu.cn
Purpose: Caregivers of COPD patients experience various caregiving burden, which is related to their quality of life. We aimed to explicitly explore whether social support and negative coping styles play the chain mediating roles in the relationship between the caregiving burden of caregivers of COPD patients and their health-related quality of life.

Methods: We conducted a multicenter cross-sectional survey in four hospitals using a convenience sample. Participants who met the inclusion criteria completed five relevant scales, including a sociodemographic scale. One-way ANOVA and Pearson's method were used to analyze the data accordingly, and chain mediated effect values were estimated by bootstrap method to determine whether the model holds.

Results: Among the 201 caregivers who participated in this study, the mean age was 55.94 years, most of them were female $(61.2 \%)$, the mean caregiving burden score was $52.39 \pm$ 14.65 , and the quality of life score was $37.97 \pm 3.55$. Among them, age, gender, education, relationship with the patient, and the number of chronic diseases were the main factors affecting the quality of life score of the caregivers. According to Pearson results, there was a negative correlation between quality of life and caregiving burden, and negative coping styles. The model result showed that caregiving burden could directly and negatively predict quality of life, and also predict quality of life through the chain mediation of social support and negative coping styles.

Conclusion: In China, caregivers of patients with COPD experience severe levels of caregiving burden and low quality of life. The combination of adequate perceived social support and lower negative coping can be effective in alleviating caregiving burden and improving their quality of life. Therefore, healthcare professionals should provide targeted guidance to caregivers with the help of social support and interventions that regulate negative coping styles.

Keywords: COPD, caregiving burden, quality of life, social support, negative coping styles, cross-sectional study

\section{Introduction}

Chronic Obstructive Pulmonary Disease (COPD) is a common chronic respiratory disease characterized by persistent airflow limitation and respiratory symptoms in middle-aged and elderly patients in the community. ${ }^{1}$ According to $\mathrm{WHO}$, about 3.04 million people died from COPD worldwide in 2016, accounting for $5.3 \%$ of all global deaths that year. The number of deaths from COPD is expected to increase to 
7.05 million per year by $2060 .^{2}$ According to epidemiological surveys in China, the percentage of COPD patients among people over 40 years of age is as high as $8.6 \%$, and there are about 99.9 million adults with COPD in China, which is a very large number, far exceeding the national population of many countries. ${ }^{3}$ COPD is now the third leading cause of death in China, and it is reported that only $2.6 \%$ of respondents in China are aware that they have the disease. ${ }^{4}$ COPD is now a major global public health challenge due to its high morbidity, disability and mortality rates and low awareness rates. ${ }^{5}$

Patients with COPD are often accompanied by numerous other diagnosed or underlying chronic diseases such as cardiovascular disease, depression and osteoporosis, which can have a serious negative impact on their health status. ${ }^{6}$ Many studies have shown that the disease characteristics of COPD predispose patients to recurrent disease, prolonged disease duration, and long-term treatment. ${ }^{7}$ As a result, most patients experience a progressive decline in their ability to live independently on a daily basis, making them progressively less able to care for themselves and increasingly dependent on their caregivers, which invariably creates a caregiving burden for their caregivers. ${ }^{8,9}$ Caregiving burden is actually a dynamically changing stressor, a subjective perception of fluctuations in patient needs and changes in the objective environment during the caregiving process. ${ }^{10}$ Properly assessing the level of caregiving burden will facilitates better COPD disease management outcomes for patients.

Previous literature suggests that caregiving burden typically occurs when caregivers face significant role shifts and begin to assume responsibility for managing the full range of patient needs. This may have a negative impact on caregivers' physical and mental health, resulting in a significant decline in their own quality of life. This suggests that the level of caregiver burden is closely related to quality of life. ${ }^{11,12}$ Quality of life is often considered a culturally dependent, multidimensional and complex concept that includes physical, psychological, social and material dimensions of life. ${ }^{13}$ Understanding the current quality of life of caregivers of people with COPD and the factors that influence it, as well as strategies for its improvement, is an important health and social priority.

Social support is often considered a protective factor in reducing caregiver burden and improving their quality of life. ${ }^{14}$ Existing research points out that fewer caregivers of COPD patients perceive adequate social support and that their level of social support utilization is not high. ${ }^{15}$
Perceived social support refers to the degree to which individuals perceive the evaluation and expectations of the full range of social support they receive. ${ }^{16}$ Social support as a process of interpersonal interaction not only brings practical help and provides emotional communication to caregivers but also effectively reduces the severity of stress. ${ }^{17}$ According to Coyne's psychological stress theory, social support and coping styles play an important role in buffering individual stress processes, and the caregiver's caregiving burden was used as a source of individual stress in this study. ${ }^{18}$ Perceived social support is also effective in negatively predicting caregiver burden. ${ }^{14}$ It has also been suggested that the level of social support is an important predictor of caregiver quality of life, and the higher the level of perceived social support, the higher the level of quality of life of the individual. ${ }^{19}$

Caregivers who respond negatively when providing care to patients may create a vicious cycle effect with their poor mental health status. ${ }^{20}$ Trait coping style is a relatively stable behavioral and cognitive approach taken by individuals in the face of various stressful events, and includes both positive and negative coping styles. ${ }^{21}$ Previous studies have shown that if caregivers' negative coping styles are not improved in the process of caring for patients, it will seriously affect the caregivers' social adjustment, increase the patients' adverse psychological stress and caregiving burden, as well as lead to their functional deficits and reduced quality of life level in the long run. ${ }^{21,22}$ It was found that caregivers' negative coping styles should be moderated more than positive coping styles. This is because negative coping can have many negative effects on caregivers in the daily life of longterm care, but is easily intervened. ${ }^{23,24}$ Instead, most of the current research is stuck on overall coping styles, and very few studies have been conducted to explore the mechanisms of negative coping and adaptive interventions in a targeted manner.

However, most previous studies have focused on the study of the relationship between caregiver burden and quality of life, and significantly less attention has been paid to the role of social support and negative coping styles in the caregiver population of COPD patients. ${ }^{25}$ What's more, the mechanisms of how these two mediating variables affect quality of life based on burden of care have not been elucidated, meaning that the pathways between the factors are unclear. Based on this, the aim of this study was to investigate the relationship between burden of care and quality of life among caregivers of 
patients with COPD, and to explore whether both social support and negative coping act as chain mediators in this relationship. Based on this, this study aimed to investigate the current status of caregiving burden and quality of life of COPD caregivers, to explore the chain mediating relationship between social support and negative coping, and to provide strong theoretical support for future improvement of caregivers' quality of life.

\section{Materials and Methods}

\section{Study Design, Setting, and Participants}

This study was a cross-sectional survey. Caregivers of COPD inpatients in four large tertiary hospitals $(>2000$ beds) in Shandong Province from December 2019 to June 2020 were selected as the study population using a convenience sampling method, and the participants were given paper questionnaires and instructed to fill them out.

The inclusion criteria for caregivers were as follows: (i) the patients cared for should meet the diagnostic criteria of the Global Initiative for Chronic Obstructive Lung Disease $(G O L D)^{26}$ and have been diagnosed for $\geq 6$ months; (ii) the caregivers were family members who provided the most core care to the patients without receiving any remuneration; (iii) the caregivers had been caring for the patients for $\geq 3$ months cumulatively; and (iv) the caregivers were $\geq 18$ years of age.

Exclusion criteria were as follows: (i) the caregiver has a serious physical illness, such as malignancy or intellectual-psychiatric problems; and (ii) the caregiver is unwilling to participate or asks to withdraw in the middle of the process.

\section{Procedures}

After signing an informed consent form, primary caregivers of COPD patients participated in our on-site paperand-pencil survey during the patients' hospitalization. The survey was administered in the conference room or seminar room of the respiratory medicine wards of the four hospitals. The scales to be completed were designed to understand the caregiver's burden of care, social support, negative coping styles, health-related quality of life, and demographic characteristics of the participants.

The research team was mainly postgraduate students majoring nursing, and the team received a week of relevant training before conducting the study, which included the research background, purpose, and significance of the survey. They were responsible for recruiting study participants, distributing questionnaires, and helping participants who had difficulty reading the questionnaires.

A total of 250 caregivers were invited to participate in this study by telephone contact or e-mail, of which 216 agreed to participate (Response rate: 86.4\%) and 216 questionnaires were collected finally. Our team assessed the completeness of the questionnaires collected on the same day, and all the questionnaires were handed over to the team members who were trained in questionnaire management for safekeeping. After our evaluation, there were 201 valid completed questionnaires (Effective rate: $93.1 \%)$.

\section{Ethical Considerations}

This study was conducted in compliance with the Declaration of Helsinki, ${ }^{27}$ and was approved by the Ethics Committee of the College of Nursing and Rehabilitation, Shandong University (No. 2021-R-0312). Study participants were informed of the purpose and process of the study prior to participation and were informed of their right to withdraw from the study at any time. The nature, benefits, uses, and adverse effects of this study were explained to all of them. Written informed consent was obtained from all participants prior to the start of the study.

\section{Measurement}

\section{Independent Variable: Caregiving Burden}

We assessed the caregiving burden of caregivers by Zarit Caregiving Burden interview (ZBI) which was designed by Zarit et al. ${ }^{28}$ The scale is a Likert 5-point scale with 22 items, and its total score is 88 . The lower the score indicated the lower caregiving burden. This scale has been widely used in China with good reliability and validity, ${ }^{29}$ and its Cronbach's alpha coefficient in this study was 0.879 .

\section{Mediator I: Perceived Social Support}

We used the Perceived social support Scale (PSSS) which was developed by Zimet et $\mathrm{al}^{30}$ and translated by Jiang et $\mathrm{al}^{31}$ to measure caregivers' perceived social support. The PSSS consists of two components, intrafamilial and extrafamilial support, and has 12 items scored on a Likert 7-point scale (1=strongly disagree, increasing in steps to $7=$ extremely agree). The total scale scores ranged from 12 to 84 , with total intra-family support scores ranging from 4 to 28 and total extra-family support scores ranging from 8 to 56 . The 
lower the score means the worse the social support perceived by the caregiver. The Chinese version of the scale was confirmed to have good reliability, ${ }^{32}$ and the Cronbach's alpha coefficient in this study was 0.952 .

\section{Mediator 2: Negative Coping Styles}

Trait Coping Style Questionnaire (TCSQ) is mainly used to assess the individual's personal characteristics and has an impact on their health status. ${ }^{33}$ The scale consists of 2 dimensions, positive coping styles and negative coping styles, containing a total of 20 items, and is scored on a Likert 5 scale. The subscale of negative coping styles (TCSQ-NCS) was selected as the instrument for this study to understand the degree of negative coping of caregivers. The Chinese version of the subscale was proved to have good applicability, and the Cronbach's alpha coefficient of the negative coping subscale in this study was 0.806 .

\section{Dependent Variable: Health-Related Quality of Life} The Version II of Mos 12-items Short Form Health Survey (SF-12 v2) is a simplified version of the 36item Brief Health Questionnaire Version II, which developed by Ware et $\mathrm{al}^{34}$ and is now widely used to measure the quality of life of caregivers. Its Chinese version has been proven to have good reliability and validity. ${ }^{35}$ This scale includes 8 dimensions of physical functioning, physical functioning, somatic pain, general health status, vitality, social functioning, emotional functioning, and psychological health. Based on the above 8 dimensions, the total physical health and total mental health of the caregivers can be further calculated to represent their quality of life at the somatic and psychological levels, respectively. The Cronbach's alpha coefficient of the scale in this study was 0.874 .

\section{Demographic Characteristics}

This questionnaire was designed independently by the researchers with full reference to the literature review and group discussions. It contained information to be completed by the participants such as age, gender, marital status, monthly income, relationship with the patient, education background (primary, secondary, college and above), place of residence (rural, urban), cumulative years of care, number of chronic diseases suffered and average time spent in care per day.

\section{Statistical Analyses}

Descriptive analyses of demographic information were used to test the effect of variables with different demographic information on quality of life scores using one-way ANOVA. Pearson correlation analysis was performed on four variables (caregiving burden, social support, negative coping, and quality of life) using SPSS version 24.0 software. To test the significance of the chained mediation model in this study, we used the SPSS process macro model 6 $(2013)^{36}$ to operation. This method is based on ordinary least squares regression and Bootstrap methods to estimate the significance of confidence intervals for mediated effects, and the corresponding effect values are statistically significant if the interval does not contain $0 . P<0.05$ was considered to indicate a statistically significant difference. Process macros were implemented using one independent variable (burden of care), two mediating variables (social support and negative coping styles), and one dependent variable (quality of life). In addition, we used age, gender, education and number of chronic diseases suffered as covariates in this chain-mediating model.

\section{Results}

\section{Participant Characteristics}

We collected data on a total of 201 caregivers accompanying COPD patients during their hospitalization. The age range of the caregivers in this study was between 30 and 82 , caregivers were predominantly female $(61.2 \%)$, mostly at less than college educational background $(78.1 \%)$, and caregivers were mostly children of patients $(65.7 \%)$, and the cumulative years of care range from 2 to 40 years, see Table 1 . According to the results of the analysis, the burden of care score of caregivers of COPD patients was $52.39 \pm 14.65$. Their overall quality-of-life score was $37.97 \pm 3.55$, which was lower than that of the Chinese normative population. ${ }^{37}$ The perceived social support score of participants was $66.25 \pm 15.11$, and their negative coping style score was $24.45 \pm 9.06$, see Table 2 .

One-way ANOVA results showed that caregivers' current quality of life scores differed in terms of age $(F=4.775, P=0.009)$, gender $(F=1.007, P=0.001)$, education $(F=3.490, P=0.032)$, caregiver-patient relationship $(F=12.932, P=0.000)$, and number of chronic diseases suffered ( $F=6.979, P=0.001)$ were statistically significant.

\section{Pearson Correlation Analysis}

As shown in Table 2, Pearson correlation analysis showed a negative correlation between caregiving burden and 
Table I Descriptive Analysis of Demographic Data, Scores for Burden of Care and Quality of Life

\begin{tabular}{|c|c|c|c|}
\hline Category & Subcategory & $\mathbf{N}(\%)$ & Quality of Life Mean (SD) \\
\hline \multirow[t]{3}{*}{ Age } & $\leq 44$ & $33(16.4 \%)$ & $38.88 \pm 2.87$ \\
\hline & $44 \sim 59$ & $82(40.8 \%)$ & $38.51 \pm 3.37$ \\
\hline & $\geq 60$ & $86(42.8 \%)$ & $37.10 \pm 3.78$ \\
\hline $\mathrm{F}$ & & & 4.775 \\
\hline P-value & & & 0.009 \\
\hline \multirow[t]{2}{*}{ Gender } & Male & $78(38.8 \%)$ & $38.96 \pm 3.35$ \\
\hline & Female & $123(61.2 \%)$ & $37.34 \pm 3.54$ \\
\hline $\mathrm{F}$ & & & 1.007 \\
\hline P-value & & & 0.001 \\
\hline \multirow[t]{2}{*}{ Residence } & Rural & $53(26.4 \%)$ & $37.96 \pm 3.66$ \\
\hline & Urban area & $148(73.6 \%)$ & $37.97 \pm 3.52$ \\
\hline $\mathrm{F}$ & & & 0.002 \\
\hline P-value & & & 0.985 \\
\hline \multirow[t]{3}{*}{ Education } & Primary School & $45(22.4 \%)$ & $36.93 \pm 4.02$ \\
\hline & Secondary School & $\mathrm{II} 2(55.7 \%)$ & $38.03 \pm 3.29$ \\
\hline & College or above & $44(21.9 \%)$ & $38.89 \pm 3.46$ \\
\hline $\mathrm{F}$ & & & 3.490 \\
\hline P-value & & & 0.032 \\
\hline \multirow[t]{3}{*}{ Income per month } & $\leq 4000$ & $90(44.8 \%)$ & $37.76 \pm 3.89$ \\
\hline & $4000-7000$ & $95(47.2 \%)$ & $37.94 \pm 3.03$ \\
\hline & $\geq 7000$ & $16(8.0 \%)$ & $39.31 \pm 4.25$ \\
\hline $\mathrm{F}$ & & & 1.317 \\
\hline P-value & & & 0.270 \\
\hline \multirow[t]{3}{*}{ Relationship with patient } & Spouse & $67(33.3 \%)$ & $36.31 \pm 3.64$ \\
\hline & Children & $132(65.7 \%)$ & $38.78 \pm 3.21$ \\
\hline & Grandchildren & $2(1 \%)$ & $41.50 \pm 2.12$ \\
\hline $\mathrm{F}$ & & & 12.932 \\
\hline P-value & & & 0.000 \\
\hline \multirow[t]{3}{*}{ Cumulative years of care } & $<5$ years & $88(43.8 \%)$ & $38.22 \pm 3.41$ \\
\hline & $5-10$ years & $56(27.9 \%)$ & $37.91 \pm 3.41$ \\
\hline & $\geq 10$ years & $57(28.4 \%)$ & $37.65 \pm 3.90$ \\
\hline $\mathrm{F}$ & & & 0.450 \\
\hline P-value & & & 0.638 \\
\hline \multirow[t]{3}{*}{ Number of chronic diseases suffered } & None & $71(35.3 \%)$ & $38.86 \pm 3.17$ \\
\hline & I to 3 & $103(51.2 \%)$ & $37.88 \pm 3.63$ \\
\hline & More than 3 & $27(13.4 \%)$ & $35.96 \pm 3.41$ \\
\hline $\mathrm{F}$ & & & 6.979 \\
\hline P-value & & & 0.001 \\
\hline
\end{tabular}


Table 2 Pearson Correlations Between the Four Variables of Caregiving Burden, Social Support, Negative Coping, and Quality of Life

\begin{tabular}{|l|c|c|c|c|c|}
\hline Variables & MD \pm SD & I & $\mathbf{2}$ & $\mathbf{3}$ & $\mathbf{4}$ \\
\hline I. Caregiving burden & $52.39 \pm 14.65$ & I & - & - & - \\
2. Quality of life & $37.97 \pm 3.55$ & $-0.330^{* *}$ & 1 & - & - \\
3. Social support & $66.24 \pm 14.26$ & $-0.218^{* *}$ & $0.149^{*}$ & 1 & - \\
4. Negative coping style & $24.45 \pm 9.06$ & $0.394^{* *}$ & $-0.263^{* *}$ & $-0.488^{* *}$ & I \\
\hline
\end{tabular}

Notes: $* \mathrm{P}<0.05, * * \mathrm{P}<0.01$.

quality of life $(r=-0.330, P<0.01)$, social support $(r=$ $-0.218, P<0.01)$ and a positive correlation with negative coping style $(r=0.394, P<0.01)$. Quality of life and social support were positively correlated $(r=0.149, P<0.05)$ and negatively correlated with negative coping $(r=-0.263$, $P<0.01)$. The correlation coefficients indicated that the effect sizes between the variables ranged from -0.1 to 0.1 , and the $p$-values for the correlations between the variables were statistically significant.

\section{Analysis of Chain Mediation Effects}

Four variables that were significantly correlated were modeled as chain mediators. A bias-corrected percentile Bootstrap (5000 replicate samples) was used to determine the role of social support and negative coping in mediating the chain variables between caregiver's burden of care and quality of life. The standardized path coefficients were calculated to reduce Type 1 errors due to distribution (Hayes, 2013), and the results of the chain mediation model analysis are shown in Figure 1.

The total effect of caregiving burden on quality of life (c=0.211, $P<0.01)$ was statistically significant, and the direct effect of path was $(\mathrm{c}=0.372, P<0.01)$. Caregiving burden had a negative direct effect on social support $(\beta=$ $-0.218, P<0.01)$ and a positive direct effect on negative coping style $(\beta=0.302, P<0.01)$. Also, social support as a first mediating variable had a significant negative direct effect on negative coping style second mediating variable $(\beta=-0.422, P<0.01)$

As shown in Table 3, when the variables of caregiver's age, gender, education, relationship with the patient, as well as the number of chronic diseases suffered were included as covariates in the model, the paths through the single mediating variable $M_{1}$ social support (point estimate $=-0.0123 ; 95 \%$ BC CI $[-0.0226,-0.0039])$, the path through two mediators (point estimate $=-0.0207 ; 95 \%$ BC CI [-0.0364, -0.0079$])$, and the path through the single mediating variable $\mathrm{M}_{2}$ negative coping style (point estimate $=-0.0185 ; 95 \% \mathrm{BC}$ CI $[-0.0395,-0.0032])$ were all statistically significant. Moreover, the total indirect effect was also statistically significant (point estimate $=$ $-0.0515 ; 95 \%$ BC CI $[-0.0941,-0.0183])$. Thus, the path through these two chained mediating variables was significant, and the path through the indirect effects of social support only and negative coping style only also held significant.

\section{Discussion}

Given the unique Chinese traditional culture, family members are the most central caregivers of COPD patients. Our

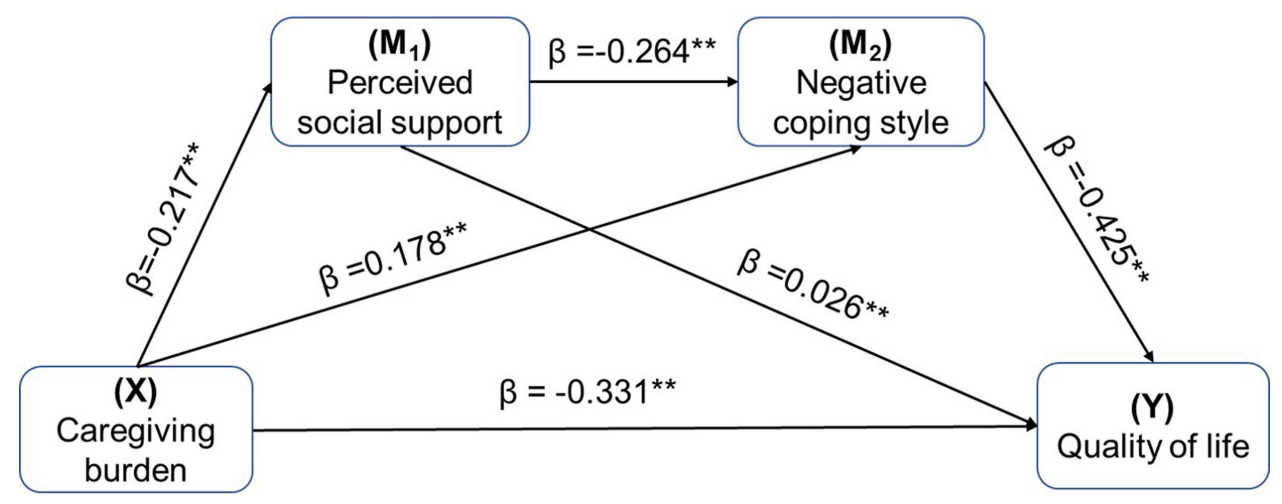

Figure I The role of social support and negative coping as chain mediators in the relationship between caregiving burden and quality of life with standardized Beta. Notes: $* * P<0.01$. Values on paths are path coefficients (standardized $\beta \mathrm{s}$ ). 
Table 3 Chain-Mediated Effects of Caregiving Burden on Quality of Life of Caregivers of COPD Patients Through Social Support and Negative Coping Styles

\begin{tabular}{|l|c|c|c|c|}
\hline \multirow{2}{*}{ Effect } & \multicolumn{2}{|c|}{ Product of Coefficients } & \multicolumn{2}{c|}{$\begin{array}{c}\text { Bootstrapping 95\% BC Confidence } \\
\text { Interval (CI) }\end{array}$} \\
\cline { 2 - 5 } & Point Estimate & Boot SE & Boot LL (CI) & Boot UL (CI) \\
\hline Total indirect effect of $X$ on $Y$ & -0.0515 & 0.0167 & -0.0941 & -0.0183 \\
Indirect effect I: $X \rightarrow M_{1} \rightarrow Y$ & -0.0123 & 0.0015 & -0.0226 & -0.0039 \\
Indirect effect 2: $X \rightarrow M_{1} \rightarrow M_{2} \rightarrow Y$ & -0.0207 & 0.0076 & -0.0364 & -0.0079 \\
Indirect effect 3: $X \rightarrow M_{2} \rightarrow Y$ & -0.0185 & 0.0093 & -0.0395 & -0.0032 \\
\hline
\end{tabular}

Notes: $N=201$. Number of bootstrap samples for bias corrected bootstrap confidence intervals: 5000 . Level of confidence for all confidence intervals: $95 \%$. $X=$ burden of care; $M_{1}=$ social support; $M_{2}=$ negative coping, and $Y=q u a l i t y$ of life. Covariates included: caregiver's age, gender, education, relationship with the patient, the number of chronic diseases suffered. Model I = caregiving burden - social support - quality of life. Model 2 = caregiving burden - social support - negative coping - quality of life. Model 3 = caregiving burden - negative coping - quality of life.

study analyzed the relationship between caregiver burden and their quality of life, while exploring the role of social support and negative coping styles on the chain of mediators that act in this relationship. The results showed that the caregiving burden score of participants was (52.39 \pm 14.65). According to the grading of the ZBI scale, it is at the heavy burden level, which results are significantly higher than those in Turkey and Portugal. ${ }^{17,38}$ Besides, the quality of life score for caregivers of patients with COPD was (37.97 \pm 3.55 ), which was significantly lower than that of caregivers of children with disabilities and patients with chronic kidney disease. ${ }^{39,40}$ The results suggest that a higher burden of care for caregivers, in turn, significantly affects their level of quality of life. The reason is that COPD patients have a long disease duration, recurrent symptoms, and progressive exacerbation, which makes their caregivers bear an unusually heavy burden of care and are prone to many physical impairments during the long-term uninterrupted care process, which has a significant negative impact on their quality of life. ${ }^{41,42}$ Vanleerberghe et $\mathrm{al}^{13}$ also found that caregiving burden also leads to a decrease in the quality of care and deterioration of the patient's condition, which in turn increases the caregiver's burden, thus creating a vicious cycle. Moreover, the influence of traditional Chinese culture on caregivers is deeply rooted and is one of the factors that cannot be ignored when interpreting the results of this study. Because of the different cultural backgrounds, the caregiving burden and quality of life levels of Chinese caregivers of COPD patients differ significantly from those of caregivers in Western countries. On the one hand, deeply influenced by thousands of years of traditional Eastern cultural teachings such as Buddhism, Taoism and Confucianism, filial piety and harmony have become the most important family values for Chinese people. ${ }^{43}$ Therefore, it is not difficult to find that in China, family members (mainly the spouse and children) are almost the core caregivers of patients, taking care of all the living, psychological care and disease management during the long disease cycle. ${ }^{44,45}$ On the other hand, the values of "Acceptance of Destiny" seem to permeate the way Chinese patients and their caregivers view health. In particular, unfortunate experiences of illness can be associated with fate, providence or luck, ie, the belief that one's health is predetermined and that little can be done to change the predetermined outcome. ${ }^{46}$ So these traditional cultures are mapped in the daily lives of Chinese caregivers, and in some ways, both of these aspects add to the caregiver's burden of caregiving on both a physical and psychological level, negatively impacting their quality of life. Therefore, there is an urgent need for a high level of attention and intervention for COPD caregivers to reduce their caregiving burden and improve their quality of life.

The present study sought to uncover potential mechanisms underlying the relationship between caregiving burden and quality of life in COPD caregivers from the perspective of a stress response model proposed by Coyne et al. ${ }^{18}$ Based on the results of the chained mediation model and comparing two pairs of specific indirect effects, we found that caregiving burden not only has a direct effect on caregivers' quality of life but also has an indirect effect on quality of life through the mediation of social support and negative coping styles. The mediation model of caregiving burden influences quality of life symptoms through three important pathways: (1) social support and negative coping styles play a significant mediating role in the relationship between caregiving burden 
and quality of life; (2) social support partially mediates the relationship between caregiving burden and quality of life; and (3) negative coping styles partially mediate the relationship between caregiving burden and quality of life. All three pathways between the relationship between caregiving burden and quality of life level among COPD caregivers were statistically significant. This important finding will help us understand the powerful role that familycentered social support and emotion regulation systems play in the daily lives of Chinese people.

Chronic caregiving stress has been previously documented to be associated with impaired endothelial cell function in caregivers, and this may be a potential mechanism leading to an increased probability of chronic underlying disease in caregivers. ${ }^{47}$ Caregivers' evaluation of caregiving burden is their subjective perception of caregiving stress. The results of this study indicate that different age, gender, education level, monthly income and number of comorbid underlying diseases are all influential factors of caregiving burden. Different caregivers often have different evaluations and tolerance of the degree of caregiving burden, and the lack of disease awareness and caregiving knowledge tends to make them more stressed and generate the perception that caregiving is difficult and burdensome, and it is this psychological perception and feeling that significantly affects their behavior and health, ${ }^{48}$ meanwhile, it also has an indelible impact on their quality of life. Consequently, healthcare professionals play an important role in guiding the process by establishing interventions aimed at improving caregivers' self-efficacy, such as teaching professional knowledge and caregiving skills and experiences of success and failure in caregiving behaviors, which will be very meaningful in reducing caregiving burden and improving their physical and mental health. ${ }^{49}$

High levels of social support have been reported to have a buffering effect on caregiver burden, helping caregivers cope with the challenges of the caregiving experience, including the impact on their physical health, mental and emotional well-being, the social isolationism, and financial stress. ${ }^{50}$ In addition, caregivers with a high level of social support will have a weakened negative coping style and will be more confident in facing difficulties, which in turn can have a protective effect on the caregiver's quality of life. ${ }^{51}$ The caregiver's physical fatigue will be greatly relieved if caregivers can receive guidance and assistance from family members or friends, and talking to family members or friends about their concerns and anxieties will provide psychological comfort and reduce negative emotions. ${ }^{52}$
Therefore, healthcare professionals who provide health education to COPD caregivers can improve the quality of life of their caregivers by conducting social support-based interventions to regulate their caregivers' negative coping styles, which can benefit the well-being of both COPD patients and caregivers, and has important implications for clinical practice.

\section{Strength and Limitations}

The strength of this study is that it is a multicenter study across four large hospitals in China with a wide coverage of the population; and it uses a validated instrument to collect data from caregivers of COPD patients. The direct and indirect pathways of the chain mediation model were also used to validate the relationships between variables.

However, this study inevitably has several limitations. First, the results of the studies are based on a crosssectional design and do not imply a causal effect of caregiving burden on quality of life. Therefore, further testing in longitudinal studies is needed to determine the association between the strength of the relationships of different variables. Second, indicators for other correlates of caregiving burden, such as caregiver competence, role conflict, and illness perception, were not measured. Follow-up more comprehensive studies are needed to investigate the effects of independent and underlying factors on caregivers' quality of life. Third, this study was conducted only in large hospitals. Both previous studies and the results of this study suggest that most COPD patients attending large tertiary hospitals have moderate and severe stages of the disease. Therefore, this study needs to be approached with caution when generalizing to all COPD patient caregiver populations.

\section{Conclusions}

This study was the first to explore the current caregiving burden of caregivers of COPD patients and the relationship between their quality of life using a chain mediated model, which showed that the caregiving burden of caregivers of COPD patients was heavy and significantly negatively correlated with their quality of life level. The chain mediated results suggest that good social support and low levels of negative coping are effective in reducing the caregiving burden of caregivers, as well as being protective factors for quality of life. Thus, health professionals should promptly assess the situation of caregiving burden of caregivers of COPD patients and screen for its main influencing factors. If necessary, integrated social support can be 
established to regulate caregivers' negative coping styles through hospital-community-family, and attention to psychological care provided to primary caregivers to alleviate their negative emotions may be crucial to reduce caregiving burden and improve quality of life levels. Factors influencing caregiving burden can be further explored in the future through high-quality longitudinal studies as a way to improve caregivers' quality of life levels and achieve higher quality of disease management for patients.

\section{Acknowledgments}

We thank all the co-authors, all the participants for their cooperation and assistance, the funds provided, Shandong University and four large general hospitals for providing the platform, and Hayes who provided the process macros for IBM SPSS 24.0. It is thanks to the above support that this study was carried out and successfully concluded.

\section{Author Contributions}

Study conceived and designed by: Mo Yi, Di Jiang, and Ou Chen.

ExperimentResearch conducted and data collected: Di Jiang, Jingjing Wang, Yuan Min Jia, and Haixia Wang.

Data analysis: Mo Yi, Di Jiang, and Wei Xu, Zeyi Zhang.

Reagents/materials/analysis tools: Yuanmin Jia and Yizhang Li.

Paper authors: Mo Yi and Ou Chen.

All authors contributed to data analysis, drafting or revising the article, have agreed on the journal to which the article will be submitted, gave final approval of the version to be published, and agree to be accountable for all aspects of the work.

\section{Funding}

This study was supported by the National Natural Science Foundation of China (81400072 to Prof CHEN Ou); the Natural Science Foundation of Shandong Province (ZR2020MH006 to Prof CHEN Ou); and the Key Research and Development Program of Shandong Province (2019GSF108198 to Prof CHEN Ou).

\section{Disclosure}

This paper has not been published elsewhere in whole or in part. All authors report no conflicts of interest for this work.

\section{References}

1. Tottenborg SS, Lange P, Johnsen SP, Nielsen H, Ingebrigtsen TS, Thomsen RW. Socioeconomic inequalities in adherence to inhaled maintenance medications and clinical prognosis of COPD. Respir Med. 2016;119:160-167. doi:10.1016/j.rmed.2016.09.007

2. World Health Organization. The top 10 causes of death. Available from: https://www.who.int/news-room/fact-sheets/detail/the-top-10causes-of-death. Accessed December 9, 2020.

3. Wang C, Xu J, Yang L, et al. Prevalence and risk factors of chronic obstructive pulmonary disease in China (the China Pulmonary Health [CPH] Study): a National Cross-Sectional Study. Lancet. 2018;391 (10131):1706-1717. doi:10.1016/S0140-6736(18)30841-9

4. Yang G, Wang Y, Zeng Y, et al. Rapid health transition in China, 19902010: findings from the Global Burden of Disease Study 2010. Lancet. 2013;381(9882):1987-2015. doi:10.1016/S0140-6736(13)61097-1

5. Fang L, Gao P, Bao H, et al. Chronic obstructive pulmonary disease in China: a Nationwide Prevalence Study. Lancet Respir Med. 2018;6 (6):421-430. doi:10.1016/S2213-2600(18)30103-6

6. Recio IJ, Diez-Manglano J, Lopez GF, Diaz PJ, Almagro P, Varela AJ. Management of the COPD patient with comorbidities: an experts recommendation document. Int $J$ Chron Obstruct Pulmon Dis. 2020;15:1015-1037.

7. Vogelmeier CF, Criner GJ, Martinez FJ, et al. Global strategy for the diagnosis, management, and prevention of chronic obstructive lung disease 2017 report. GOLD executive summary. Am J Respir Crit Care Med. 2017;195(5):557-582. doi:10.1164/rccm.201701-0218PP

8. Nakken N, Janssen DJ, van den Bogaart EH, et al. Informal caregivers of patients with COPD: home sweet home? Eur Respir Rev. 2015;24(137):498-504. doi:10.1183/16000617.00010114

9. Hsiao PC, Chu CM, Sung PY, Perng WC, Wang KY. Differences in COPD patient care by primary family caregivers: an age-based study. PLoS One. 2014;9(9):e107870. doi:10.1371/journal.pone.0107870

10. Saria MG, Courchesne NS, Evangelista L, et al. Anxiety and depression associated with burden in caregivers of patients with brain metastases. Oncol Nurs Forum. 2017;44(3):306-315. doi:10.1188/ 17.ONF.306-315

11. Choi HS, Yang DW, Rhee CK, et al. The health-related quality-of-life of chronic obstructive pulmonary disease patients and disease-related indirect burdens. Korean J Intern Med. 2020;35(5):1136-1144. doi: $10.3904 / \mathrm{kjim} .2018 .398$

12. Strang S, Fahrn J, Strang P, Ronstad A, Danielsson L. Support to informal caregivers of patients with severe chronic obstructive pulmonary disease: a qualitative study of caregivers' and professionals' experiences in Swedish hospitals. BMJ Open. 2019;9(8):e28720. doi:10.1136/bmjopen-2018-028720

13. Vanleerberghe P, De Witte N, Claes C, Schalock RL, Verte D. The quality of life of older people aging in place: a literature review. Qual Life Res. 2017;26(11):2899-2907. doi:10.1007/s11136-017-1651-0

14. Shukri M, Mustofai MA, Md YM, Tuan HT. Burden, quality of life, anxiety, and depressive symptoms among caregivers of hemodialysis patients: the role of social support. Int J Psychiatry Med. 2020;55 (6):397-407. doi:10.1177/0091217420913388

15. Chu HH, Lee KS, Ho LK, Chung JH, Shin KC, Hwang TY. Association of physical and psychological health status between chronic obstructive pulmonary disease patients and their family caregivers. Health Care Women Int. 2019;40(10):1019-1030. doi:10.1080/07399332.2019.1617292

16. Zuelsdorff ML, Koscik RL, Okonkwo OC, et al. Social support and verbal interaction are differentially associated with cognitive function in midlife and older age. Neuropsychol Dev Cogn B Aging Neuropsychol Cogn. 2019;26(2):144-160. doi:10.1080/13825585.2017.1414769

17. Goris S, Klc Z, Elmal F, Tutar N, Takc O. Care burden and social support levels of caregivers of patients with chronic obstructive pulmonary disease. Holist Nurs Pract. 2016;30(4):227-235. doi:10.1097/HNP.0000000000000153 
18. Coyne JC, Downey G. Social factors and psychopathology: stress, social support, and coping processes. Annu Rev Psychol. 1991;42 (1):401-425. doi:10.1146/annurev.ps.42.020191.002153

19. Peepratoom B, Low G, Malathum P, Chai-Aroon T, Chuchottaworn C, Arpanantikul M. A structural equation model of health-related quality of life among Thai men with chronic obstructive pulmonary disease. J Clin Nurs. 2020;29(13-14):2638-2651. doi:10.1111/jocn.15286

20. Burton AM, Sautter JM, Tulsky JA, et al. Burden and well-being among a diverse sample of cancer, congestive heart failure, and chronic obstructive pulmonary disease caregivers. J Pain Symptom Manage. 2012;44 (3):410-420. doi:10.1016/j.jpainsymman.2011.09.018

21. Han Y, Hu D, Liu Y, et al. Coping styles and social support among depressed Chinese family caregivers of patients with esophageal cancer. Eur J Oncol Nurs. 2014;18(6):571-577. doi:10.1016/j.ejon.2014.07.002

22. Hu X, Huang W, Su Y, Qu M, Peng X. Depressive symptoms in Chinese family caregivers of patients with heart failure: a Cross-Sectional Study. Medicine (Baltimore). 2017;96(13):e6480. doi:10.1097/MD.0000000000006480

23. Figueiredo D, Gabriel R, Jacome C, Marques A. Caring for people with early and advanced chronic obstructive pulmonary disease: how do family carers cope? J Clin Nurs. 2014;23(1-2):211-220. doi: 10.1111 jocn. 12363

24. Taylor SE, Stanton AL. Coping resources, coping processes, and mental health. Annu Rev Clin Psychol. 2007;3(1):377-401. doi:10.1146/annurev.clinpsy.3.022806.091520

25. Haya M, Ichikawa S, Wakabayashi H, Takemura Y. Family caregivers' perspectives for the effect of social support on their care burden and quality of life: a Mixed-Method Study in rural and suburban central Japan. Tohoku J Exp Med. 2019;247(3):197-207. doi:10.1620/tjem.247.197

26. Patel AR, Patel AR, Singh S, Singh S, Khawaja I. Global initiative for chronic obstructive lung disease: the changes made. Cureus. 2019;11(6):e4985.

27. World Medical Association. World Medical Association declaration of Helsinki: ethical principles for medical research involving human subjects. JAMA. 2013;310(20):2191-2194. doi:10.1001/ jama.2013.281053

28. Zarit SH, Todd PA, Zarit JM. Subjective burden of husbands and wives as caregivers: a Longitudinal Study. Gerontologist. 1986;26 (3):260-266. doi:10.1093/geront $/ 26.3 .260$

29. Wang M, He B, Wang Y, et al. Depression among low-income female Muslim Uyghur and Kazakh informal caregivers of disabled elders in far western china: influence on the caregivers' burden and the disabled elders' quality of life. PLoS One. 2016;11(5):e156382.

30. Zimet GD, Powell SS, Farley GK, Werkman S, Berkoff KA. Psychometric characteristics of the multidimensional scale of perceived social support. J Pers Assess. 1990;55(3-4):610-617.

31. Jiang QJ. Handbook of mental health rating scales: the Perceptual Social Support Scale (PSSS). Beijing: China Mental Health Press; 1999:131-133.

32. Yang LM, Zhouting LM, Danfeng Cao BS, Fenglin CM. Social support and depression across the perinatal period: a Longitudinal Study. J Clin Nurs. 2017;26:17-18.

33. Liu F, Zhang Z, Chen L. Mediating effect of neuroticism and negative coping style in relation to childhood psychological maltreatment and smartphone addiction among college students in China. Child Abuse Negl. 2020;106:104531. doi:10.1016/j.chiabu.2020.104531

34. Ware JE, Kosinski M, Keller SD. How to score the SF-12 physical and mental health summary scales. Boston, MA: Health Institute, New England Medical Center; 1995:11-19.

35. Liang Y, Wu W. Exploratory analysis of health-related quality of life among the empty-nest elderly in rural China: an Empirical Study in three economically developed cities in eastern China. Health Qual Life Outcomes. 2014;12(1):59. doi:10.1186/14777525-12-59
36. Preacher KJ, Hayes AF. SPSS and SAS procedures for estimating indirect effects in simple mediation models. Behav Res Methods Instrum Comput. 2004;36(4):717-731. doi:10.3758/BF03206553

37. Lam ET, Lam CL, Fong DY, Huang WW. Is the SF-12 version 2 health survey a valid and equivalent substitute for the SF-36 version 2 health survey for the Chinese? J Eval Clin Pract. 2013;19 (1):200-208. doi:10.1111/j.1365-2753.2011.01800.x

38. Hipolito N, Ruivo A, Martins S, et al. Relationship between distress related to caregiver burden and physical activity in informal caregivers of patients with COPD. Copd. 2020;17(5):562-567. doi:10.1080/15412555.2020.1799964

39. Adejumo OA, Okaka EI, Akinbodewa AA, Iyawe OI, Edeki IR, Abolarin OS. Self-perceived burden on caregivers, anxiety and depression among chronic kidney disease patients in southern Nigeria. West Afr J Med. 2021;38(4):335-341.

40. Xia C, Sun M, Li X, et al. Health-related quality of life and related factors among primary caregivers of children with disabilities in shanghai, china: a Cross-Sectional Study. Int J Environ Res Public Health. 2020;17(24):24. doi:10.3390/ijerph17249299

41. Aasbo G, Rugkasa J, Solbraekke KN, Werner A. Negotiating the care-giving role: family members' experience during critical exacerbation of COPD in Norway. Health Soc Care Community. 2017;25 (2):612-620. doi:10.1111/hsc. 12350

42. Bove DG, Zakrisson AB, Midtgaard J, Lomborg K, Overgaard D. Undefined and unpredictable responsibility: a Focus Group Study of the experiences of informal caregiver spouses of patients with severe COPD. J Clin Nurs. 2016;25(3-4):483-493. doi:10.1111/jocn.13076

43. Leung D, Chan H, Chiu P, Lo R, Lee L. Source of social support and caregiving self-efficacy on caregiver burden and patient's quality of life: a path analysis on patients with palliative care needs and their caregivers. Int $J$ Environ Res Public Health. 2020;17(15):15. doi:10.3390/ijerph17155457

44. Chan HY, Yu DS, Leung DY, Chan AW, Hui E. Quality of life and palliative care needs of elderly patients with advanced heart failure. $J$ Geriatr Cardiol. 2016;13(5):420-424.

45. Yu H, Wang X, He R, Liang R, Zhou L. Measuring the caregiver burden of caring for community-residing people with alzheimer's disease. PLoS One. 2015;10(7):e132168.

46. Liu X, Li J, Zhang Q, Zhao Y, Xu W. Being beneficial to self and caregiver: the role of dispositional mindfulness among breast cancer patients. Support Care Cancer. 2021;29(1):239-246. doi:10.1007/ s00520-020-05435-8

47. Mausbach BT, Roepke SK, Ziegler MG, et al. Association between chronic caregiving stress and impaired endothelial function in the elderly. J Am Coll Cardiol. 2010;55(23):2599-2606. doi:10.1016/j. jacc.2009.11.093

48. Chung ML, Pressler SJ, Dunbar SB, Lennie TA, Moser DK. Predictors of depressive symptoms in caregivers of patients with heart failure. J Cardiovasc Nurs. 2010;25(5):411-419. doi:10.1097/ JCN.0b013e3181d2a58d

49. Clark AM, Reid ME, Morrison CE, Capewell S, Murdoch DL, McMurray JJ. The complex nature of informal care in home-based heart failure management. $J$ Adv Nurs. 2008;61(4):373-383. doi:10.1111/j.1365-2648.2007.04527.x

50. Casado B, Sacco P. Correlates of caregiver burden among family caregivers of older Korean Americans. J Gerontol B Psychol Sci Soc Sci. 2012;67(3):331-336. doi:10.1093/geronb/gbr115

51. Nightingale CL, Curbow BA, Wingard JR, Pereira DB, Carnaby GD. Burden, quality of life, and social support in caregivers of patients undergoing radiotherapy for head and neck cancer: a pilot study. Chronic Illn. 2016;12(3):236-245. doi:10.1177/1742395316644305

52. Lindqvist G, Heikkila K, Albin B, Hjelm K. Conceptions of daily life in men living with a woman suffering from chronic obstructive pulmonary disease. Prim Health Care Res Dev. 2013;14 (2):140-150. doi:10.1017/S1463423612000394 


\section{Publish your work in this journal}

The International Journal of COPD is an international, peer-reviewed journal of therapeutics and pharmacology focusing on concise rapid reporting of clinical studies and reviews in COPD. Special focus is given to the pathophysiological processes underlying the disease, intervention programs, patient focused education, and self management

protocols. This journal is indexed on PubMed Central, MedLine and CAS. The manuscript management system is completely online and includes a very quick and fair peer-review system, which is all easy to use. Visit http://www.dovepress.com/testimonials.php to read real quotes from published authors. 